\title{
Live Demonstration: Event-Driven Sensing and Processing for High-Speed Robotic Vision
}

\author{
L. A. Camuñas-Mesa, T. Serrano-Gotarredona, and B. Linares-Barranco \\ Instituto de Microelectrónica de Sevilla (IMSE-CNM), \\ CSIC y Univ. de Sevilla, Sevilla, SPAIN \\ Email: camunas@imse-cnm.csic.es
}

\section{DEMONSTRATION SETUP}

Fig. 1(a) shows the demo setup. Two DVS boards send events out through parallel buses to a merger board. This board merges all the event flow in one single AER bus, and sends it to a custom-made convolutional board, where a 2D grid array of convolution modules is implemented within a Spartan6 FPGA, as represented in Fig. 1(b) and (c). A USBAERmini2 board is used to timestamp the events coming out of the convolutional board and send them to a computer through a high-speed USB2.0 port. Finally, the output events are represented in the computer in real time using jAER software.

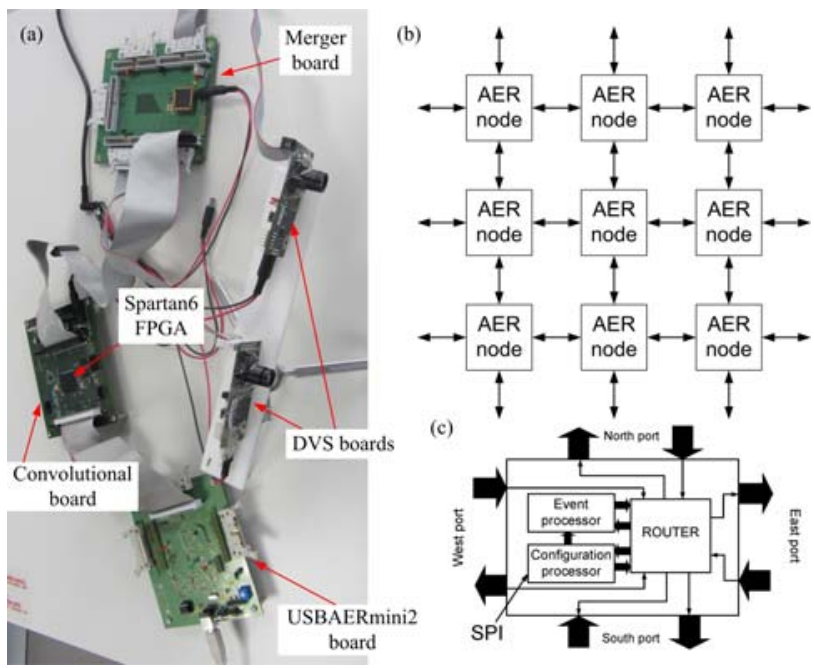

Fig. 1. (a) Example setup of a multi-sensor event-driven processing system using a Spartan6 FPGA and a family of custom-made eventhandling boards. (b) 2D network topology for multi-casting-mesh AER. (c) Diagram of AER node.

\section{VISITOR EXPERIENCE}

In this demonstration, two different experiments are proposed. In both we use visual input from one or two DVS sensors when observing a moving object, in these examples a chart containing strong vertical and horizontal patterns, as well as thin $45^{\circ}$ lines.

\section{A. Example 1}

In the first example, only one DVS is used, so the merger board is bypassed. The FPGA in the convolution board is programmed to implement a network of 3x3 Gabor filters, which process in parallel the input flow of events sent by the DVS. The kernels correspond to three different scales (kernel sizes) and three different angles $\left(0^{\circ}, 45^{\circ}\right.$ and $\left.90^{\circ}\right)$, as shown in Fig. 2(a). The simultaneous output of the 9 filters is represented in Fig. 2(c).

\section{B. Example 2}

In this case, both DVS sensors are connected to the convolution board through the merger board, adding an extra bit to each event to identify which retina produced it. Therefore, if the event is coming from the left retina, it is sent to the top three convolution module and the central left one. If the event is coming from the right retina, it is sent to the three bottom modules and the central right one. The kernels are shown in Fig. 2(b), and the output from the convolution array is illustrated in Fig. 2(d). (a) 15.
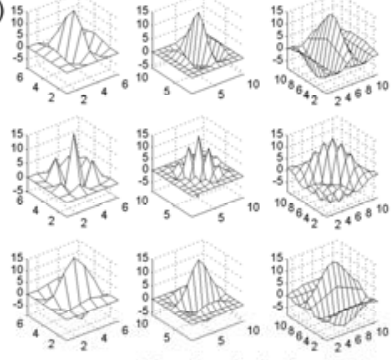

(c)

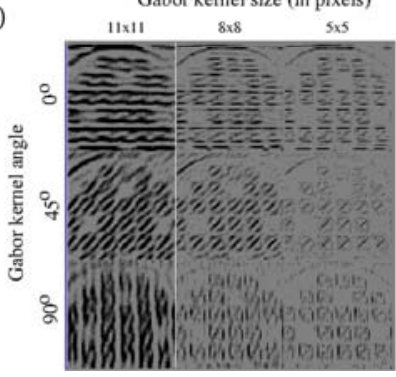

(b)
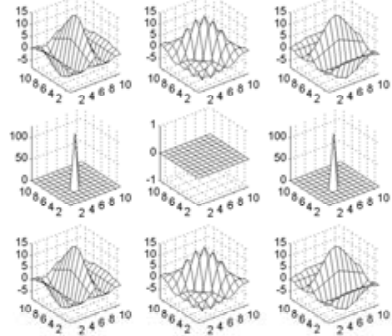

Gabor kemel angle

(d)

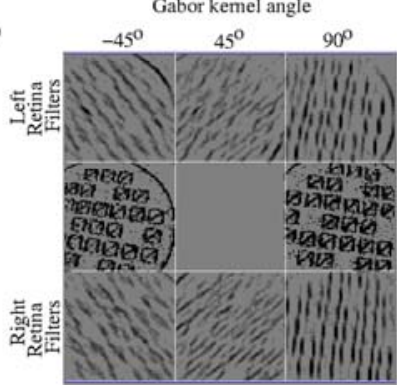

Fig. 2. Output of Event-Driven Gabor Filter Array. Example 1: one single DVS is used and events are sent to nine Gabor filters (a), processing them in parallel (c). Example 2: Stereo Vision setup: each retina output is sent to 3 Gabor filters of different angle plus another filter with unity kernel to replicate the input (b), processing the outputs of both retinas in parallel (d).

\section{EARLIER PUBLICATIONS}

[1] C. Zamarreño-Ramos et al. "Multi-Casting Mesh AER: A Scalable Assembly Approach for Reconfigurable Neuromorphic Structured AER Systems. Application to ConvNets,” IEEE Trans. on Biom. Circ. Syst., vol. 7, No. 1, pp. 82-102, Feb. 2013.

[2] L. Camuñas-Mesa et al., "On the use of orientation filters for 3D reconstruction in event-driven stereo vision,” Front. Neurosci. 8:48, 2104. 